\title{
Residues and tame symbols on toroidal varieties
}

\author{
Ivan Soprounov
}

\begin{abstract}
We introduce a new approach to the study of a system of algebraic equations in $\left(\mathbb{C}^{\times}\right)^{n}$ whose Newton polytopes have sufficiently general relative positions. Our method is based on the theory of Parshin's residues and tame symbols on toroidal varieties. It provides a uniform algebraic explanation of the recent result of Khovanskii on the product of the roots of such systems and the Gel'fond-Khovanskii result on the sum of the values of a Laurent polynomial over the roots of such systems, and extends them to the case of an algebraically closed field of arbitrary characteristic.
\end{abstract}

\section{Introduction}

1.1 The classical residue formula says that the sum of the residues of a rational 1-form $\omega$ over all points of a complex projective curve $X$ is zero:

$$
\sum_{x \in X} \operatorname{res}_{x} \omega=0
$$

The standard proof of this formula uses Stokes' theorem. In 'Algebraic groups and class fields' J.-P. Serre gives a purely algebraic proof of the residue formula which works over any algebraically closed field even of positive characteristic [Ser88].

In class field theory the residue formula has a multiplicative cousin, Weil's reciprocity law, which states that the product of the tame symbols of any two rational functions $f_{0}$ and $f_{1}$ over all points of a projective curve $X$ is one [Ser88]:

$$
\prod_{x \in X}\left\langle f_{0}, f_{1}\right\rangle_{x}=1
$$

In the 1970s A. Parshin constructed higher-dimensional class field theory where he generalized the residue and the tame symbol [Par75]. Given an $n$-dimensional algebraic variety $X$ and a rational form $\omega$ on $X$, Parshin defines the residue $\operatorname{res}_{F} \omega$ at each complete flag $F: X_{0} \subset X_{1} \subset \cdots \subset X_{n-1} \subset X$ of irreducible subvarieties of $X$. Similarly, given any $n+1$ rational functions $f_{0}, \ldots, f_{n}$ on $X$, he defines the tame symbol $\left\langle f_{0}, \ldots, f_{n}\right\rangle_{F}$ at each such flag $F$. Parshin's residue and symbol satisfy not one but many reciprocity laws: Fix all subvarieties in the flag $F$ except one, say $X_{i}$. Then the sum of the residues (product of the symbols) over all possible irreducible subvarieties $X_{i}$ that can appear in the $i$ th slot of $F$ is zero (one) (Theorem A.4 in the Appendix).

The aim of the present paper is to look at some recent results of the theory of Newton polytopes from the point of view of this general theory of Parshin. More specifically, consider a system of Laurent polynomial equations in the $n$-torus:

$$
f_{1}(t)=\cdots=f_{n}(t)=0, \quad t \in\left(\mathbb{C}^{\times}\right)^{n} .
$$

Received 23 October 2002, accepted in final form 5 November 2003, published online 15 October 2004. 2000 Mathematics Subject Classification 14M25 (primary), 52B20 (secondary).

Keywords: residue, tame symbol, combinatorial coefficients.

This journal is (C) Foundation Compositio Mathematica 2004. 


\section{Soprounov}

Suppose the Newton polytopes $\Delta_{1}, \ldots, \Delta_{n}$ of the $f_{i}$ have sufficiently general relative positions (see Definition 5.1). Then the system has a finite number of roots. O. Gel'fond and A. Khovanskii proved the following result [GK96].

Theorem A. The sum of the values of a Laurent polynomial $f_{0}$ over the roots of (1) counting multiplicities is equal to

$$
\sum_{A}(-1)^{n} c(A) \operatorname{res}_{A}\left(f_{0} \frac{d f_{1}}{f_{1}} \wedge \cdots \wedge \frac{d f_{n}}{f_{n}}\right),
$$

where the sum is over the vertices $A$ of $\Delta=\Delta_{1}+\cdots+\Delta_{n}, \operatorname{res}_{A}\left(f_{0} d f_{1} / f_{1} \wedge \cdots \wedge d f_{n} / f_{n}\right)$ is the residue at a vertex (an explicit rational function in the coefficients of the $f_{i}$ ), and $c(A)$ is the combinatorial coefficient (an integer that reflects the combinatorial structure of the polytope $\Delta$ near the vertex $A$ ).

This is, in fact, a particular case of their residue formula [GK02] for the sum of the Grothendieck residues of a rational $n$-form which is regular in $\left(\mathbb{C}^{\times}\right)^{n} \backslash\left\{f_{1} \cdots f_{n}=0\right\}$, over the roots of the system (1). The proof of the residue formula is topological and uses toric compactifications.

The following generalized Vieta formula for the product of roots of (1) has been obtained by Khovanskii [Kho99].

THEOREM B. The product of the values of a Laurent monomial $f_{0}$ over the roots of (1) counting multiplicities is equal to

$$
\prod_{A}\left[f_{0}, \ldots, f_{n}\right]_{A}^{(-1)^{n} c(A)}
$$

where the product is over the vertices $A$ of $\Delta=\Delta_{1}+\cdots+\Delta_{n},\left[f_{0}, \ldots, f_{n}\right]_{A}$ is the symbol at a vertex (an explicit Laurent monomial in the coefficients of the $f_{i}$ ), and $c(A)$ is the combinatorial coefficient.

The proof of this theorem uses the polyhedral homotopy method and regular subdivisions of polytopes.

The relation between Theorems A and B appears to be the same as the one between the onedimensional residue formula and Weil's reciprocity. Moreover, the number $\left[f_{0}, \ldots, f_{n}\right]_{A}$ is defined similarly to Parshin's tame symbol. This gives a motivation to search for a uniform explanation of these results in terms of the theory of residues and tame symbols.

The main obstruction to this is the notion of combinatorial coefficients $c(A)$ since they are defined as the local degrees of certain real non-algebraic maps. In the present paper we give an explicit algebraic description for the combinatorial coefficients as a signed number of certain complete flags of faces of $\Delta$, thus putting them in the framework of Parshin's theory. (A similar description of the combinatorial coefficient was obtained by O. Gel'fond [Gel96] for some special collections of polytopes.) We provide a uniform algebraic proof of Theorems A and B based on Parshin's theory for toroidal varieties. We also extend them to the case of an arbitrary algebraically closed field.

1.2 The material of the paper is organized as follows. In $\S 2$ we give an explicit formula for the degree of a map of polyhedral sets defined by some combinatorial data. As an application we obtain a new formula for the combinatorial coefficient.

In $\S \S 3$ and 4 we consider Parshin's theory for toroidal pairs. A toroidal pair $(X, D)$ consists of a normal variety $X$ and a codimension-1 subvariety $D$ such that, locally at each point, $X$ is analytically isomorphic to an affine toric variety $X_{\sigma}$ where the branches of $D$ correspond to the invariant divisors of $X_{\sigma}$. We define residue and tame symbol at each point $x \in X$ for which the corresponding affine variety $X_{\sigma}$ has a zero-dimensional orbit. This generalizes the notions of residue and symbol at a vertex from Theorems A and B. Our definition is similar to the one 


\section{RESIDUES AND TAME SYMBOLS ON TOROIDAL VARIETIES}

of Parshin, but does not involve any particular choice of a complete flag. Using the algebraic description of the combinatorial coefficients we prove general results about symbol and residue on toroidal pairs (Theorems 3.15 and 4.8). In $\S 5$ we show how these results imply Theorems A and $\mathrm{B}$ for arbitrary algebraically closed fields.

Finally, in the Appendix we include the definition of Parshin's residue and tame symbol and formulate the higher-dimensional reciprocity laws.

\subsection{Remarks}

There is a topological construction for the tame symbol based on Deligne's proof of Weil's reciprocity (see [BM96]). This construction provides a uniform topological explanation of the product of roots formula and the residue formula. Our approach is algebraic and works over algebraically closed fields of arbitrary characteristic.

A different formula for the product of the roots of a system (1) can be derived from Poisson's formula for the mixed resultant due to Pedersen and Sturmfels [PS93]. In this formula the product of the values of a monomial $f_{0}=c t^{m}$ over the roots is represented by the product of the mixed resultant of $f_{0}, \ldots, f_{n}$ (which in this case is just $c$ to the power of the mixed volume of $\Delta_{1}, \ldots, \Delta_{n}$ ) and the facet resultants to certain powers. The assumption on the Newton polytopes of (1) implies that each facet resultant is a monomial in one of the coefficients of the system. To give an idea of how this formula is related to the one in Theorem $\mathrm{B}$, let us assume that $f_{0}=c$, for $c \neq 0,1$. Then Poisson's formula gives the inductive formula for the mixed volume (e.g. see [BZ88, p. 166]), whereas Theorem B gives Khovanskii's formula for the mixed volume in terms of combinatorial coefficients [Kho99].

Parshin's residue is closely related to the toric residue defined by D. Cox [Cox96]. For different applications of residues in toric geometry we refer the reader to works of E. Cattani, D. Cox, A. Dickenstein, and B. Sturmfels [CCD97, CD97, CDS98].

1.4 In this paper $k$ is always an algebraically closed field. A variety is a reduced separated scheme of finite type over $k$; a subvariety is a reduced subscheme. By $\mathbb{T}$ we denote the algebraic $n$-torus over $k, \mathbb{T}=\left(k^{\times}\right)^{n}$, and $M=\operatorname{Hom}_{\text {alg.gp }}\left(\mathbb{T}, k^{\times}\right)$the abelian rank- $n$ group of characters of $\mathbb{T}$. Finally, $X_{\sigma}$ denotes the affine toric variety Spec $k[\sigma \cap M]$ defined by a convex rational polyhedral cone $\sigma$ in $M \otimes \mathbb{R}$.

\section{Degree of polyhedral maps and combinatorial coefficient}

In this section we show how to compute the degree of a map between two polyhedral sets which is defined by a map of the partially ordered sets of their faces. As an application we obtain an explicit combinatorial formula for the combinatorial coefficient.

\subsection{Polyhedral maps}

A polyhedral set is a finite union of convex compact polytopes intersecting in faces. We will assume that all the polytopes are embedded in a Euclidean space $E$ of some big dimension. Then a polyhedral set is a topological space with the topology inherited from $E$. The dimension of a polyhedral set is the maximum of dimensions of the polytopes it contains. A polyhedral set is oriented if every polytope it contains is oriented.

Let $X$ be a polyhedral set and $\mathcal{F}(X)$ the set of all faces of all polytopes appearing in $X$. The set $\mathcal{F}(X)$ is a finite partially ordered set by inclusion.

Consider two polyhedral sets $X$ and $Y$, and fix a map $\psi: \mathcal{F}(X) \rightarrow \mathcal{F}(Y)$ that preserves the partial ordering. A continuous piecewise linear map $f_{\psi}: X \rightarrow Y$ is called a polyhedral map associated with $\psi$ if $f_{\psi}(G) \subset \psi(G)$ for every face $G \in \mathcal{F}(X)$. 


\section{Soprounov}

Given any map $\psi: \mathcal{F}(X) \rightarrow \mathcal{F}(Y)$ there exists a polyhedral map $f_{\psi}: X \rightarrow Y$. Moreover all such maps are homotopy equivalent within the class of all polyhedral maps associated with $\psi$.

Proposition 2.1. Each map $\psi: \mathcal{F}(X) \rightarrow \mathcal{F}(Y)$ that respects the partial ordering defines a homotopy class of polyhedral maps $f_{\psi}: X \rightarrow Y$ associated with $\psi$.

Proof. First, for every map of partially ordered sets $\psi: \mathcal{F}(X) \rightarrow \mathcal{F}(Y)$ we construct a continuous piecewise linear map $f_{\psi}: X \rightarrow Y$ as follows.

Fix barycentric subdivisions of $X$ and $Y$. Note that for any polyhedral set $X$ there is a oneto-one correspondence between the set of all simplices in a barycentric subdivision of $X$ and the set of all chains in $\mathcal{F}(X)$. Consider a $k$-simplex $\Delta^{k}$ in the subdivision of $X$. It corresponds to a chain $X_{0} \subset \cdots \subset X_{k}$ in $\mathcal{F}(X)$. Let $\psi\left(X_{0}\right) \subseteq \cdots \subseteq \psi\left(X_{k}\right)$ be its image. It corresponds to a unique simplex (possibly of smaller dimension) in the subdivision of $Y$, the simplex being denoted by $\psi\left(\Delta^{k}\right)$. Since there is a unique linear map between two simplices that maps vertices of one simplex to the prescribed vertices of the other simplex, we get a map $f_{\psi}: X \rightarrow Y$ that sends each simplex $\Delta^{k}$ to the corresponding simplex $\psi\left(\Delta^{k}\right)$. Clearly this map agrees on the common faces of simplices of the subdivision and, hence, is continuous piecewise linear. By construction, $f_{\psi}(G) \subset \psi(G)$ for any $G \in \mathcal{F}(X)$.

Now suppose $f_{\psi}$ and $f_{\psi}^{\prime}$ are two polyhedral maps associated with $\psi$. Then for each $0 \leqslant t \leqslant 1$ the map $f_{\psi}^{t}=(1-t) f_{\psi}+t f_{\psi}^{\prime}$ is also associated with $\psi$. Indeed, fix a face $G \in \mathcal{F}(X)$. Then every point $x \in G$ is mapped to a point $f_{\psi}^{t}(x)$ on the segment joining $f_{\psi}(x)$ and $f_{\psi}^{\prime}(x)$. Since both $f_{\psi}(x)$ and $f_{\psi}^{\prime}(x)$ belong to the face $\psi(G)$, then $f_{\psi}^{t}(x)$ also does. Therefore $f_{\psi}^{t}(G) \subset \psi(G)$.

\subsection{Flags and degree of polyhedral maps}

Consider an oriented polyhedral set $X$. Let $\mathcal{X}: X_{0} \subset \cdots \subset X_{n}, \operatorname{dim} X_{i}=i$ be a complete flag in $X$, i.e. a maximal chain of elements of $\mathcal{F}(X)$. With the flag $\mathcal{X}$ we associate an ordered set of vectors $\left(e_{1}, \ldots, e_{n}\right)$, where $e_{i}$ begins at $X_{0}$ and points strictly inside $X_{i}$. Define the sign of $\mathcal{X}$ to be 1 if $\left(e_{1}, \ldots, e_{n}\right)$ gives a positive oriented frame for $X_{n}$ and -1 otherwise. It is easy to see that the sign does not depend on the choice of vectors $e_{1}, \ldots, e_{n}$. We denote it by $\operatorname{sgn} \mathcal{X}$.

Now consider a map of partially ordered sets $\psi: \mathcal{F}(X) \rightarrow \mathcal{F}(Y)$, where $X$ and $Y$ are $n$-dimensional oriented polyhedral sets. Every polyhedral map $f_{\psi}: X \rightarrow Y$ associated with $\psi$ induces a map of the $n$th homology groups:

$$
H_{n}\left(f_{\psi}\right): H_{n}(X) \rightarrow H_{n}(Y) .
$$

By Proposition 2.1 this map is the same for all choices of $f_{\psi}$. We call it the degree map of $\psi$. We will be concerned with the case when both groups $H_{n}(X)$ and $H_{n}(Y)$ are isomorphic to $\mathbb{Z}$. (This is true, for example, when $X$ and $Y$ are the boundaries of $(n+1)$-dimensional polytopes.) Then the degree map is the multiplication by an integer, which we denote by $\operatorname{deg}(\psi)$. In the next theorem we show how to compute $\operatorname{deg}(\psi)$ as a signed number of certain complete flags in $X$.

Let $\mathcal{X}: X_{0} \subset \cdots \subset X_{n}$ and $\mathcal{Y}: Y_{0} \subset \cdots \subset Y_{n}$ be complete flags in $X$ and $Y$, respectively. We will write $\psi(\mathcal{X})=\mathcal{Y}$ if and only if $\psi\left(X_{i}\right)=Y_{i}$ for all $0 \leqslant i \leqslant n$. Define the preimage of $\mathcal{Y}$ under $\psi$ to be the set of all $\mathcal{X}$ such that $\psi(\mathcal{X})=\mathcal{Y}$.

Theorem 2.2. Let $X$ and $Y$ be two polyhedral sets as above, and $\psi: \mathcal{F}(X) \rightarrow \mathcal{F}(Y)$ a map of partially ordered sets of their faces. Fix any complete flag $\mathcal{Y}$ in $Y$. Then the degree of $\psi$ is equal to the sign of $\mathcal{Y}$ times the signed number of all complete flags $\mathcal{X}$ in $X$ in the preimage of $\mathcal{Y}$ under $\psi$ :

$$
\operatorname{deg}(\psi)=\operatorname{sgn} \mathcal{Y} \sum_{\psi(\mathcal{X})=\mathcal{Y}} \operatorname{sgn} \mathcal{X}
$$




\section{RESIDUES AND TAME SYMBOLS ON TOROIDAL VARIETIES}

Proof. By Proposition 2.1 we can choose any function in the homotopy class defined by $\psi$. We take $f_{\psi}$ to be the piecewise linear function constructed in the proof of Proposition $2.1 \mathrm{using}$ barycentric subdivisions of $X$ and $Y$. We view $f_{\psi}$ as a simplicial map between two simplicial complexes.

Fix any positive oriented $n$-simplex $\Delta_{Y}^{n}$ in the barycentric subdivision of $Y$. Then the degree of $f_{\psi}$ is the number of all $n$-simplices $\Delta_{X}^{n}$ in $X$ that are mapped to $\Delta_{Y}^{n}$; each simplex being counted with either sign plus or sign minus according to its orientation. Recall that the $n$-simplex $\Delta_{Y}^{n}$ corresponds to a complete flag $\mathcal{Y}$ in $Y$ and every $n$-simplex $\Delta_{X}^{n}$ corresponds to some complete flag $\mathcal{X}$ in $X$. Clearly, the orientation of $\Delta_{X}^{n}$ coincides with the sign of the corresponding flag $\mathcal{X}$, and $f_{\psi}\left(\Delta_{X}^{n}\right)=\Delta_{Y}^{n}$ if and only if $\psi(\mathcal{X})=\mathcal{Y}$. It remains to notice that if we fix a negative oriented $\Delta_{Y}^{n}$ then the number we obtain is the negative degree of $f_{\psi}$.

\subsection{Combinatorial coefficient}

The combinatorial coefficient is a local analog of the degree considered above.

Let $\sigma \subset \mathbb{R}^{n}$ be a convex polyhedral $n$-dimensional cone with apex $A$. Consider an ordered collection $D=\left(D_{1}, \ldots, D_{m}\right)$ of $m$ distinct non-empty closed subsets of $\sigma$, where $m \leqslant n$ and each set $D_{i}$ is a union of facets of $\sigma$. Assume that they cover the boundary of $\sigma$ and, if $m=n$, that the apex $A$ is the only face of $\sigma$ which is covered by all of them:

$$
\partial \sigma=D_{1} \cup \cdots \cup D_{m}, \quad \text { if } m=n \text { then } D_{1} \cap \cdots \cap D_{n}=\{A\} .
$$

A continuous map $g: \sigma \rightarrow \mathbb{R}^{n}$ is called a characteristic map of the covering (2) if for each $i$, $1 \leqslant i \leqslant n$, the $i$ th component $g_{i}$ of $g$ is non-negative and vanishes precisely on those faces of $\sigma$ that belong to $D_{i}$. It is easy to see that all characteristic maps send the boundary of $\sigma$ to the boundary of the positive octant $\mathbb{R}_{+}^{n}$ such that $g^{-1}(0) \subseteq\{A\}$, and they are homotopy equivalent within the class of such maps.

Definition 2.3. The local degree of the germ at $A$ of the restriction of a characteristic map to the boundary of $\sigma$,

$$
\bar{g}:(\partial \sigma, A) \rightarrow\left(\partial \mathbb{R}_{+}^{n}, 0\right),
$$

is called the combinatorial coefficient of the covering (2).

Clearly, the combinatorial coefficient is zero unless $m=n$. In the case when $m=n$, Theorem 2.2 provides us with a description of the combinatorial coefficient as the number of certain complete flags of faces of $\sigma$, counted with signs.

For a cone $\sigma \subset \mathbb{R}^{n}$ we let $\mathcal{F}(\partial \sigma)$ denote the partially ordered by inclusion set of the proper faces of $\sigma$. With a covering (2) we associate a map $\phi: \mathcal{F}(\partial \sigma) \rightarrow \mathcal{F}\left(\partial \mathbb{R}_{+}^{n}\right)$ by putting

$$
\phi(\tau)=\mathbb{R}_{+}^{n} \cap\left\{y_{i_{1}}=\cdots=y_{i_{k}}=0\right\}
$$

if and only if $\tau$ is a common face of $D_{i_{1}}, \ldots D_{i_{k}}$ for $1 \leqslant i_{l} \leqslant n$, and $k$ is maximal. Here $\left(y_{1}, \ldots, y_{n}\right)$ is a coordinate system for $\mathbb{R}^{n}$.

For any complete flag $\gamma_{0} \subset \cdots \subset \gamma_{n-1}$ in $\partial \mathbb{R}_{+}^{n}$ define its preimage under $\phi$ as the set of all complete flags $\sigma_{0} \subset \cdots \subset \sigma_{n-1}$ in $\partial \sigma$ such that $\phi\left(\sigma_{i}\right)=\gamma_{i}, 0 \leqslant i \leqslant n-1$. Note that the preimage of any flag under $\phi$ is empty if $m<n$.

Fix the standard orientation of $\mathbb{R}^{n}$. We orient the boundary of every $n$-dimensional cone in $\mathbb{R}^{n}$ in accordance with this fixed orientation. As before, define the sign of a complete flag $\sigma_{0} \subset \cdots \subset \sigma_{n-1}$ to be 1 if it gives a positive oriented frame for $\sigma_{n-1}$, and -1 otherwise.

THEOREM 2.4. The combinatorial coefficient of a covering (2) is equal to the signed number of all complete flags in the preimage of any positive complete flag under $\phi$. 


\section{Soprounov}

In particular, if $m=n$ the combinatorial coefficient is equal to the signed number of all complete flags $\sigma_{0} \subset \cdots \subset \sigma_{n-1} \subset \sigma$, where $\sigma_{i}$ is a common face of $D_{i+1}, \ldots, D_{n}$ of dimension $i$.

Proof. The case $m<n$ is obvious, so we assume that $m=n$. To be able to apply Theorem 2.2 we 'compactify' the cones $\sigma$ and $\mathbb{R}_{+}^{n}$. Consider a pyramid with the vertex $A$ and base $D_{0}$ which is a cross-section of $\sigma$ by a generic hyperplane. Let $X$ be the oriented boundary of the pyramid. Next consider the standard $n$-dimensional simplex defined in $\mathbb{R}^{n}$ as the convex hull of the origin and the endpoints of the standard basis vectors. Let $Y$ be its oriented boundary.

The subsets $D_{1}, \ldots, D_{n}$ of $\sigma$ along with the base $D_{0}$ form a covering of $X$. Define the map $\psi: \mathcal{F}(X) \rightarrow \mathcal{F}(Y)$, by putting $\psi(G)=\left\{y_{i_{1}}=\cdots=y_{i_{k}}=0\right\}$ if and only if $G$ is a common face of $D_{i_{1}}, \ldots, D_{i_{k}}$ for $0 \leqslant i_{l} \leqslant n$, and $k$ is maximal. Here $\left(y_{0}, y_{1}, \ldots, y_{n}\right)$ are the barycentric coordinates for the simplex.

Note that the restriction of a characteristic map $g: \sigma \rightarrow \mathbb{R}^{n}$ to $X$ defines a polyhedral map $f_{\psi}: X \rightarrow Y$ associated with $\psi$. According to Theorem 2.2, the degree of $\psi$ is equal to the number of complete flags of faces of $X$ counted with signs in the preimage under $\psi$ of any positive complete flag of faces of $Y$. For example, one can take the flag

$$
\left\{y_{1}=\cdots=y_{n}=0\right\} \subset\left\{y_{2}=\cdots=y_{n}=0\right\} \subset \cdots \subset\left\{y_{n}=0\right\} .
$$

Remark 2.5. Notice that since there are $n$ ! complete flags in $\mathbb{R}_{+}^{n}$ we obtain $n$ ! formulae for the combinatorial coefficient. If $m=n$ a choice of a complete flag corresponds to an order of $D_{1}, \ldots, D_{n}$, and thus we can say that the combinatorial coefficient is skew-symmetric in $D_{1}, \ldots, D_{n}$.

\section{Toroidal symbol}

The toroidal symbol is a slight modification of Parshin's tame symbol for toroidal varieties. More precisely, consider a pair $(X, D)$ consisting of a normal variety $X$ and a codimension- 1 subset $D$ such that, in a formal neighborhood of each point, $X$ is isomorphic to an affine toric variety $X_{\sigma}$ and $D$ corresponds to the invariant divisor $X_{\sigma} \backslash \mathbb{T}$. We distinguish special points on $X$ for which the corresponding toric variety has a zero-dimensional orbit. At each such point $x \in X$ the toroidal symbol associates a non-zero element $\left[f_{0}, \ldots, f_{n}\right]_{x}$ of the base field to every collection of $n+1$ rational functions $f_{0}, \ldots, f_{n}$ on $X$ with divisors in $D$.

Suppose the irreducible components of $D$ are divided into $2 n$ groups $D_{1}^{\prime}, \ldots, D_{n}^{\prime}, D_{1}^{\prime \prime}, \ldots, D_{n}^{\prime \prime}$ (where $n=\operatorname{dim} X$ ) and assume that the sets $S^{\prime}=D_{1}^{\prime} \cap \cdots \cap D_{n}^{\prime}$ and $S^{\prime \prime}=D_{1}^{\prime \prime} \cap \cdots \cap D_{n}^{\prime \prime}$ consist of special points only. The main result of this section is a certain reciprocity between the products of symbols over $S^{\prime}$ and $S^{\prime \prime}$.

\subsection{Toroidal pair}

Here we recall the definition of a toroidal pair. A detailed treatment of toroidal pairs is given in [KKMS73] where they are called toroidal embeddings without self-intersections. We use Danilov's terminology from [Dan78].

Let $X$ be a normal $n$-dimensional variety over an algebraically closed field $k$. Let $D$ be a closed subset of $X$, every irreducible component of which is a codimension-1 normal subvariety of $X$. We say that the pair $(X, D)$ is toroidal if for every closed point $x \in X$ there exists an $n$-dimensional algebraic torus $\mathbb{T}$, an affine toric variety $X_{\sigma}$ (corresponding to a rational convex $n$-dimensional cone $\sigma)$, and a point $x_{0}$ in $X_{\sigma}$ such that $(X, D, x)$ is formally locally isomorphic to $\left(X_{\sigma}, X_{\sigma} \backslash \mathbb{T}, x_{0}\right)$. The latter means that there exists an isomorphism of the formal completions of the local rings

$$
\widehat{\mathcal{O}}_{X, x} \cong \widehat{\mathcal{O}}_{X_{\sigma}, x_{0}}
$$




\section{RESIDUES AND TAME SYMBOLS ON TOROIDAL VARIETIES}

such that the image of the ideal of $D$ is mapped to the image of the ideal of $X_{\sigma} \backslash \mathbb{T}$. We call $\left(X_{\sigma}, x_{0}\right)$ a local model of $(X, D)$ at $x$.

Consider the $n$-form

$$
\omega_{0}=\frac{d t_{1}}{t_{1}} \wedge \cdots \wedge \frac{d t_{n}}{t_{n}}
$$

where $\left(t_{1}, \ldots, t_{n}\right)$ are coordinates in $\mathbb{T}$. Automorphisms of $\mathbb{T}$ correspond to monomial changes of coordinates

$$
u_{i}=t_{1}^{q_{i 1}} \ldots t_{n}^{q_{i n}}, \quad 1 \leqslant i \leqslant n, \quad Q=\left(q_{i j}\right) \in G L(n, \mathbb{Z}) .
$$

We will write $u=t^{Q}$ to denote the monomial change of coordinates (3). Note that the form $\omega_{0}$ is preserved under monomial changes of coordinates with $\operatorname{det} Q=1$, and changes the sign when $\operatorname{det} Q=-1$. Therefore, $\omega_{0}$ provides an analog of orientation on $X_{\sigma}$.

Furthermore, the choice of coordinates in $\mathbb{T}$ defines an orientation of the space of characters $M \otimes \mathbb{R}$. Monomial changes of coordinates (3) preserve this orientation if and only if $\operatorname{det} Q=1$. Therefore, the orientation of $M \otimes \mathbb{R}$ and hence of $\sigma$ is uniquely determined by the form $\omega_{0}$.

We call $\left(X_{\sigma}, x_{0}, \omega_{0}\right)$ an equipped local model of $(X, D)$ at $x$, assuming that the form $\omega_{0}$ is fixed and the cone $\sigma$ is oriented accordingly.

Let $D=\bigcup_{i \in I} E_{i}$ be the decomposition of $D$ into irreducible components. The components of the sets $\bigcap_{i \in J} E_{i} \backslash \bigcup_{i \notin J} E_{i}$ (where $J \subset I$ ) are non-singular and define a stratification of $X$ (see [KKMS73, p. 57]). In particular, $X \backslash D$ is non-singular. The components of $\bigcap_{i \in J} E_{i}$ are normal and are the closures of the strata. Furthermore, for each $x \in X$ the closures of strata which contain $x$ correspond formally to the closures of the orbits in a local model $\left(X_{\sigma}, x_{0}\right)$ at $x$.

We denote by $\mathrm{St}_{i}(X)$ the set of all $i$-dimensional strata, and by $\overline{\mathrm{St}}_{i}(X)$ the set of the closures of the $i$-dimensional strata. Note that if $x \in \operatorname{St}_{0}(X)$ then in every local model $\left(X_{\sigma}, x_{0}\right)$ at $x$ the cone $\sigma$ has an apex and $x_{0}$ is the closed orbit in $X_{\sigma}$.

In the next proposition we describe what coordinate transformations relate different local models at a point $x \in \operatorname{St}_{0}(X)$.

Proposition 3.1. Let $(X, D)$ be a toroidal pair, $x \in \mathrm{St}_{0}(X)$. Then for any two local models $\left(X_{\sigma}, x_{0}\right)$ and $\left(X_{\sigma^{\prime}}, x_{0}^{\prime}\right)$ at $x$, every isomorphism

$$
\pi: \widehat{\mathcal{O}}_{X_{\sigma}, x_{0}} \cong \widehat{\mathcal{O}}_{X_{\sigma^{\prime}}, x_{0}^{\prime}}
$$

that maps the image of the ideal of $X_{\sigma} \backslash \mathbb{T}$ to the image of the ideal of $X_{\sigma^{\prime}} \backslash \mathbb{T}^{\prime}$ is induced by a change of coordinates of the form

$$
u_{i}=\phi_{i} t_{1}^{q_{i 1}} \ldots t_{n}^{q_{i n}}, \quad \phi_{i} \in \widehat{\mathcal{O}}_{X_{\sigma}, x_{0}}^{\times}, \quad 1 \leqslant i \leqslant n, \quad Q=\left(q_{i j}\right) \in G L(n, \mathbb{Z}),
$$

where $t_{1}, \ldots, t_{n}$ and $u_{1}, \ldots, u_{n}$ are coordinate functions on the tori $\mathbb{T}$ and $\mathbb{T}^{\prime}$, respectively.

Proof. Let $\Sigma(x)$ be the union of all strata $Z$ whose closure $\bar{Z}$ contains $x$. Denote by $M(x)$ the group of the Cartier divisors on $\Sigma(x)$, supported on $\Sigma(x) \cap D$, and by $M(x)_{+}$the subsemigroup of effective divisors. For each local model $\left(X_{\sigma}, x_{0}\right)$ at $x, M(x)$ is canonically isomorphic to the group of characters $M$ of $X_{\sigma}$, and $M(x)_{+}$is canonically isomorphic to the semigroup $\sigma \cap M$ (see [KKMS73, p. 61]). Therefore the semigroups $\sigma \cap M$ and $\sigma^{\prime} \cap M^{\prime}$ are isomorphic. In coordinates $t_{1}, \ldots, t_{n}$ and $u_{1}, \ldots, u_{n}$, the isomorphism corresponds to a monomial transformation $u=t^{Q}$, for $Q \in G L(n, \mathbb{Z})$.

To describe all isomorphisms $\pi: \widehat{\mathcal{O}}_{X_{\sigma}, x_{0}} \cong \widehat{\mathcal{O}}_{X_{\sigma^{\prime}}, x_{0}^{\prime}}$ it suffices to describe all automorphisms $\alpha$ of $\widehat{\mathcal{O}}_{X_{\sigma}, x_{0}}$ that fix the orbits of $X_{\sigma}$. Let $t_{1}, \ldots, t_{n}$ be coordinates in $\mathbb{T}$. Then the ring $\widehat{\mathcal{O}}_{X_{\sigma}, x_{0}}$ can be identified with the ring of all formal power series in $t_{1}, \ldots, t_{n}$ supported in $\sigma \cap M$, where $M$ is identified with $\mathbb{Z}^{n}$. Denote this ring by $A$. Let $S$ be a multiplicative subset of $A$ consisting of all elements $\phi t^{a}$, where $a \in \sigma \cap M$ and $\phi$ is an invertible element of $A$. Then for every automorphism $\alpha$, 


\section{Soprounov}

we have $\alpha(S) \subseteq S$. Indeed, since $\alpha$ fixes the orbits of $X_{\sigma}$, it maps every ideal $\left(t^{a}\right)$ to itself. Thus $\alpha\left(t^{a}\right)=\phi t^{a}$, for some invertible $\phi$. Therefore, $\alpha$ induces an automorphism $\alpha_{S}$ of the localization $A_{S}$. Note that $t_{1}, \ldots, t_{n} \in A_{S}$, since the elements of $\sigma \cap M$ generate $M$ as a group. Therefore, for each $i, 1 \leqslant i \leqslant n, \alpha_{S}\left(t_{i}\right)=\phi_{i} t_{i}$ for some invertible $\phi_{i}$.

Conversely, every map $t_{i} \mapsto \phi_{i} t_{i}, 1 \leqslant i \leqslant n, \phi_{i} \in A^{\times}$, defines an automorphism $\alpha$ of $A$, which fixes the orbits. Indeed, for every element $f \in A, f=\sum_{a} \lambda_{a} t^{a}, a \in \sigma \cap M$, put

$$
\alpha(f)=\sum_{a \in \sigma \cap M} \lambda_{a} \phi^{a} t^{a}, \quad \phi^{a}=\phi_{1}^{a_{1}} \ldots \phi_{n}^{a_{n}} .
$$

Note that the coefficient of each monomial $t^{b}$ of the series (4) is defined by finitely many series $\lambda_{a} \phi^{a} t^{a}$ (this is true since the cone $\sigma$ has an apex). Therefore this is a well-defined power series. Since all the monomials in the series belong to the semigroup $\sigma \cap M$, the series defines an element of $A$. It is easy to check that $\alpha$ is in fact a homomorphism. Also it is clearly invertible.

\subsection{Covering and combinatorial coefficients}

Definition 3.2. Let $(X, D)$ be a toroidal pair. We say that $\left(D_{1}, \ldots, D_{n}\right)$ is a reasonable covering of $D$ if $D=D_{1} \cup \cdots \cup D_{n}$, where each $D_{i}$ is the union of some irreducible components of $D$, and $D_{1} \cap \cdots \cap D_{n} \subseteq \operatorname{St}_{0}(X)$.

Let $\left(D_{1}, \ldots, D_{n}\right)$ be a reasonable covering of $D$. Consider an equipped local model $\left(X_{\sigma}, x_{0}, \omega_{0}\right)$ at a point $x \in \operatorname{St}_{0}(X)$. It can be easily seen that the covering $\left(D_{1}, \ldots, D_{n}\right)$ defines a covering of the boundary of the cone $\sigma$ in the sense of (2). This allows us to define the combinatorial coefficient of the covering $\left(D_{1}, \ldots, D_{n}\right)$ at each point $x \in \operatorname{St}_{0}(X)$.

Definition 3.3. The combinatorial coefficient at $x \in \operatorname{St}_{0}(X)$ of the covering $\left(D_{1}, \ldots, D_{n}\right)$ is the combinatorial coefficient of the induced covering of $\sigma$ in an equipped local model at $x$. We denote it by $c(x)$.

Remark 3.4 (Invariance). By Remark 2.5 the combinatorial coefficient is the same for any two equipped local models that correspond to an automorphism of $\mathbb{T}$ that preserves the form $\omega_{0}$, and changes sign otherwise. Also it is skew-symmetric in $D_{1}, \ldots, D_{n}$.

Now consider the stratification defined by the irreducible components of $D$ (see $\S 3.1$ ) and let $F$ be a complete flag of stratum closures on $X$ :

$$
F: X_{0} \subset X_{1} \subset \cdots \subset X_{n-1} \subset X, \quad X_{i} \in \overline{\operatorname{St}}_{i}(X) .
$$

It corresponds to a complete flag of orbit closures in an equipped local model $\left(X_{\sigma}, x_{0}, \omega_{0}\right)$ of $(X, D)$ at $X_{0}$, hence to a complete flag $F_{\sigma}$ of faces of $\sigma$.

Definition 3.5. We say that the flag $F$ is positive (respectively negative) and write $\operatorname{sgn} F=1$ (respectively $\operatorname{sgn} F=-1$ ) if the induced flag $F_{\sigma}$ of faces of $\sigma$ is positive (respectively negative).

Like in the case of the combinatorial coefficient, the sign of the flag depends on the choice of the form $\omega_{0}$ in an equipped local model.

Definition 3.6. Let $Z$ be a stratum. We say that the closure $\bar{Z}$ has signature $\left\{i_{1}, \ldots, i_{k}\right\}$ for $1 \leqslant i_{l} \leqslant n$ if and only if $Z \subseteq D_{i_{1}} \cap \cdots \cap D_{i_{k}}$ and $k$ is maximal.

The following proposition is the analog of the description of the combinatorial coefficient given in Theorem 2.4. 


\section{RESIDUES AND TAME SYMBOLS ON TOROIDAL VARIETIES}

Proposition 3.7. Let $(X, D)$ be toroidal and $\left(D_{1}, \ldots, D_{n}\right)$ a reasonable covering of $D$. Then the combinatorial coefficient $c(x)$ of $x \in \mathrm{St}_{0}(X)$ is equal to the number of all complete flags

$$
x=X_{0} \subset X_{1} \subset \cdots \subset X_{n-1} \subset X,
$$

where $X_{i} \in \overline{\operatorname{St}}_{i}(X)$ is a stratum closure of signature $\{i+1, \ldots, n\}, 0 \leqslant i \leqslant n-1$, counting signs.

\subsection{Symbol of monomials}

DEFINITION 3.8. Consider an ordered collection of $n+1$ monomials in $n$ variables with coefficients in a field $k$ :

$$
c_{i} t^{a_{i}}=c_{i} t_{1}^{a_{i 1}} \ldots t_{n}^{a_{i n}}, \quad c_{i} \in k^{\times}, \quad a_{i}=\left(a_{i 1}, \ldots, a_{i n}\right) \in \mathbb{Z}^{n}, \quad 0 \leqslant i \leqslant n .
$$

Let $A=\left(a_{i j}\right) \in M_{n+1, n}(\mathbb{Z})$ be the matrix whose rows are the vectors of exponents $a_{i}$. Then the symbol of $n+1$ monomials is the non-zero element of $k$ defined by

$$
\left[c_{0} t^{a_{0}}, \ldots, c_{n} t^{a_{n}}\right]=(-1)^{B} \prod_{i=0}^{n} c_{i}^{(-1)^{i} A_{i}},
$$

where $A_{i}$ is the determinant of the matrix obtained from $A$ by eliminating its $i$ th row, and

$$
B=\sum_{k} \sum_{i<j} a_{i k} a_{j k} A_{i j}^{k}
$$

where $A_{i j}^{k}$ is the determinant of the matrix obtained from $A$ by eliminating its $i$ th and $j$ th rows and its $k$ th column.

Proposition 3.9. Let $f_{i}=c_{i} t^{a_{i}}, 0 \leqslant i \leqslant n$, be monomials. The symbol has the following properties:

i) Multiplicativity. Suppose $f_{i}$ is a product of two monomials $f_{i}=f_{i}^{\prime} f_{i}^{\prime \prime}$. Then

$$
\left[f_{0}, \ldots, f_{i}^{\prime} f_{i}^{\prime \prime}, \ldots, f_{n}\right]=\left[f_{0}, \ldots, f_{i}^{\prime}, \ldots, f_{n}\right]\left[f_{0}, \ldots, f_{i}^{\prime \prime}, \ldots, f_{n}\right] .
$$

ii) Multiplicative skew-symmetry.

$$
\left[f_{0}, \ldots, f_{i}, \ldots, f_{j}, \ldots, f_{n}\right]=\left[f_{0}, \ldots, f_{j}, \ldots, f_{i}, \ldots, f_{n}\right]^{-1} .
$$

iii) Invariance.

a) Let $u=t^{Q}, Q \in G L(n, \mathbb{Z})$ be a monomial change of coordinates. Then

$$
\left[\bar{f}_{0}, \ldots, \bar{f}_{n}\right]=\left[f_{0}, \ldots, f_{n}\right]^{\operatorname{det} Q},
$$

where $\bar{f}_{i}=c_{i} u^{a_{i}}=c_{i} t^{a_{i} Q}$ and $f_{i}=c_{i} t^{a_{i}}, 0 \leqslant i \leqslant n$.

b) Let $s=\lambda t$ be a translation, i.e. $s_{j}=\lambda_{j} t_{j}, \lambda_{j} \in k^{\times}, 1 \leqslant j \leqslant n$. Then

$$
\left[f_{0}^{\prime}, \ldots, f_{n}^{\prime}\right]=\left[f_{0}, \ldots, f_{n}\right],
$$

where $f_{i}^{\prime}=c_{i} s^{a_{i}}=c_{i} \lambda^{a_{i}} t^{a_{i}}$ and $f_{i}=c_{i} t^{a_{i}}, 0 \leqslant i \leqslant n$.

Proof. Modulo the sign $(-1)^{B}$ all the properties follow easily from the properties of the determinant.

To take care of the sign we give an invariant description of $B$, following [Kho99]. Consider $B$ as a $\mathbb{Z} / 2 \mathbb{Z}$-valued function of the rows $a_{0}, \ldots, a_{n}$ of the matrix $A$. It is easy to see that $B=B\left(a_{0}, \ldots, a_{n}\right)$ is multilinear and its value on each collection of $n+1$ standard vectors $\left(e_{i_{0}}, \ldots, e_{i_{n}}\right)$ is 0 if more than two of the vectors $e_{i_{0}}, \ldots, e_{i_{n}}$ coincide; and 1 otherwise.

Now define the function $B^{\prime}=B^{\prime}\left(a_{0}, \ldots, a_{n}\right)$ to be 0 if the rank of $\left(a_{0}, \ldots, a_{n}\right)$ is less than $n$; and $\lambda_{0}+\cdots+\lambda_{n}+1(\bmod 2)$ if the vectors $a_{0}, \ldots, a_{n}$ satisfy a (unique) non-trivial relation $\lambda_{0} a_{0}+$ $\cdots+\lambda_{n} a_{n}=0$. The function $B^{\prime}$ is multilinear and on each collection $\left(e_{i_{0}}, \ldots, e_{i_{n}}\right)$ the functions $B^{\prime}$ and $B$ take the same value. Therefore $B=B^{\prime}$; in particular, $B$ is symmetric and invariant under non-degenerate transformations. 


\section{SOPROUNOV}

\subsection{Toroidal symbol}

Let $(X, D)$ be toroidal. Let $k(X, D)$ denote the set of rational functions on $X$ whose divisor lies in $D$.

Consider a zero-dimensional stratum $x \in \operatorname{St}_{0}(X)$. Then the image of $f \in k(X, D)$ in an equipped local model $\left(X_{\sigma}, x_{0}, \omega_{0}\right)$ at $x$ is the product of a monomial $c t^{a}$ and a regular invertible function $\phi \in \widehat{\mathcal{O}}_{X_{\sigma}, x_{0}}^{\times}$with $\phi\left(x_{0}\right)=1$. We call this monomial the leading monomial of $f$ at $x$. The leading monomial is defined up to monomial transformations.

Definition 3.10. Let $(X, D)$ be toroidal and $x \in \mathrm{St}_{0}(X)$ a zero-dimensional stratum. Define the toroidal symbol $\left[f_{0}, \ldots, f_{n}\right]_{x}$ at $x$ of $f_{0}, \ldots, f_{n} \in k(X, D)$ to be the symbol of the leading monomials of $f_{0}, \ldots, f_{n}$ at $x$.

Remark 3.11 (Invariance). Let $\left(X_{\sigma^{\prime}}, x_{0}^{\prime}, \omega_{0}^{\prime}\right)$ and $\left(X_{\sigma^{\prime \prime}}, x_{0}^{\prime \prime}, \omega_{0}^{\prime \prime}\right)$ be two equipped local models at $x$. Let $f^{\prime}$ and $f^{\prime \prime}$ be the images of $f \in k(X, D)$ in the two equipped local models. Then, according to Proposition 3.1, the leading monomials of $f^{\prime}$ and $f^{\prime \prime}$ are related by a composition of a monomial transformation and a translation: $t \mapsto \lambda t^{Q}$. Therefore, by Proposition 3.9 the toroidal symbol is the same for the two equipped local models if $\operatorname{det} Q=1$, and is reciprocal otherwise.

By Proposition 3.9 the toroidal symbol is multiplicative and multiplicatively skew-symmetric in $f_{0}, \ldots, f_{n}$.

Now we will give a relation between the toroidal symbol and Parshin's tame symbol at a complete flag of stratum closures on $X$.

Proposition 3.12. Let $(X, D)$ be toroidal. Consider $n+1$ rational functions $f_{0}, \ldots, f_{n} \in k(X, D)$. Then, for any complete flag $F: X_{0} \subset X_{1} \subset \cdots \subset X_{n-1} \subset X$ of stratum closures on $X$, we have

$$
\left\langle f_{0}, \ldots, f_{n}\right\rangle_{F}=\left[f_{0}, \ldots, f_{n}\right]_{X_{0}}^{\operatorname{sgn} F}
$$

where $\left\langle f_{0}, \ldots, f_{n}\right\rangle_{F}$ denotes Parshin's tame symbol at the flag $F$.

(Note that the number $\left[f_{0}, \ldots, f_{n}\right]_{X_{0}}^{\operatorname{sgn} F}$ is already independent of the choice of $\omega_{0}$ in an equipped local model.)

Proof. Since the definitions of the toroidal symbol and Parshin's tame symbol are local, we can pass to an equipped local model $\left(X_{\sigma}, x_{0}, \omega_{0}\right)$ at $X_{0}$ and assume that $F$ is a complete flag of orbit closures in $X_{\sigma}$.

Let $F_{\sigma}$ be the complete flag of faces of $\sigma$ corresponding to $F$,

$$
F_{\sigma}: \quad 0=\sigma_{0} \subset \sigma_{1} \subset \cdots \subset \sigma_{n-1} \subset \sigma_{n}=\sigma .
$$

Fix coordinates $\left(t_{1}, \ldots, t_{n}\right)$ in $\mathbb{T}, M \cong \mathbb{Z}^{n}$. Inside each $\sigma_{i}, 1 \leqslant i \leqslant n$, choose a lattice point $q_{i} \in \mathbb{Z}^{n}$ at lattice distance 1 from $\sigma_{i-1}$. Let $u_{i}=t^{q_{i}}$ be a monomial change of coordinates in $\mathbb{T}$. Then the rational functions $u_{i}=t^{q_{i}}$ give a system of local parameters at $F$ (see Appendix). Therefore by Proposition 3.9 and Remark A.2 of the Appendix,

$$
\left\langle f_{0}, \ldots, f_{n}\right\rangle_{F}=\left[c_{0} u^{k_{0}}, \ldots, c_{n} u^{k_{n}}\right]=\left[c_{0} t^{k_{0}}, \ldots, c_{n} t^{k_{n}}\right]^{\operatorname{det} Q},
$$

where $Q=\left(q_{1}, \ldots, q_{n}\right)$ and $c_{i} t^{k_{i}}$ is the leading monomial of $f_{i}$. It remains to note that $\operatorname{det} Q=$ $\operatorname{sgn} F$.

Corollary 3.13. Let $(X, D)$ be toroidal and $\left(D_{1}, \ldots, D_{n}\right)$ be a reasonable covering of $D$. For $x \in \operatorname{St}_{0}(X)$ let $\mathcal{F}(x)$ be the set of all complete flags

$$
x=X_{0} \subset X_{1} \subset \cdots \subset X_{n-1} \subset X,
$$

where $X_{i} \in \overline{\operatorname{St}}_{i}(X)$ is a stratum closure of signature $\{i+1, \ldots, n\}, 0 \leqslant i \leqslant n-1$. 


\section{RESIDUES AND TAME SYMBOLS ON TOROIDAL VARIETIES}

Then for any $n+1$ rational functions $f_{0}, \ldots, f_{n} \in k(X, D)$ we have

$$
\left[f_{0}, \ldots, f_{n}\right]_{x}^{c(x)}=\prod_{F \in \mathcal{F}(x)}\left\langle f_{0}, \ldots, f_{n}\right\rangle_{F},
$$

where we assume that the product is 1 if $\mathcal{F}(x)$ is empty.

Proof. This follows from Propositions 3.7 and 3.12 .

Remark 3.14. Note that for a toroidal pair $(X, D)$ all Parshin's reciprocity laws (Theorem A.4) for $i>0$ follow from Proposition 3.12. Indeed, consider a complete flag $F: X_{0} \subset \cdots \subset X_{i} \subset \cdots \subset X_{n}$ of irreducible subvarieties of $X$. We can pass to a local model at $X_{0}$ and assume that $X$ is an affine toric variety and $D=X \backslash \mathbb{T}$. Then for any $n+1$ rational functions $f_{0}, \ldots, f_{n} \in k(X, D)$ the symbol $\left\langle f_{0}, \ldots, f_{n}\right\rangle_{F}$ is trivial unless $F$ is a flag of orbit closures on $X$. But if we fix all $X_{j}, j \neq i$, and vary $X_{i}$, there are only two such flags $F$ (since for any face $\tau$ of a polyhedral cone there are only two codimension- 1 faces of $\tau$ that contain a fixed codimension-2 face of $\tau$ ), and the signs of these two flags are opposite. Now we can apply Proposition 3.12.

\subsection{Main theorem}

TheOrem 3.15. Let $X$ be a complete normal $n$-dimensional variety over an algebraically closed field $k$, and $D$ a closed subset of $X$ such that the pair $(X, D)$ is toroidal.

Let $\left(D_{1}, \ldots, D_{n}\right)$ be a reasonable covering of $D$ such that each $D_{i}$ is a disjoint union of two closed subsets of pure codimension 1:

$$
D=D_{1} \cup \cdots \cup D_{n}, \quad D_{1} \cap \cdots \cap D_{n} \subseteq \mathrm{St}_{0}(X), \quad D_{i}=D_{i}^{\prime} \sqcup D_{i}^{\prime \prime}, \quad 1 \leqslant i \leqslant n .
$$

We get $2^{n}$ disjoint finite closed subsets of $X$ :

$$
S_{k}=G_{1} \cap \cdots \cap G_{n} \text {, where } G_{i}=D_{i}^{\prime} \text { or } D_{i}^{\prime \prime}, \quad 1 \leqslant i \leqslant n, \quad 1 \leqslant k \leqslant 2^{n} .
$$

Then for any $n+1$ rational functions $f_{0}, \ldots, f_{n} \in k(X, D)$, the following $2^{n}$ numbers are equal:

$$
\left(\prod_{x \in S_{1}}\left[f_{0}, \ldots, f_{n}\right]_{x}^{c(x)}\right)^{(-1)^{\left|S_{1}\right|}}=\cdots=\left(\prod_{x \in S_{2^{n}}}\left[f_{0}, \ldots, f_{n}\right]_{x}^{c(x)}\right)^{(-1)^{\left|S_{2} n\right|}},
$$

where $\left[f_{0}, \ldots, f_{n}\right]_{x}$ is the toroidal symbol of $f_{0}, \ldots, f_{n}$ at $x, c(x)$ is the combinatorial coefficient at $x$, and $\left|S_{k}\right|$ is the number of $D_{i}^{\prime \prime}$ in the definition of $S_{k}$.

Proof. Because of the symmetry it is sufficient to prove the equality for any two sets

$$
S_{1}=D_{1}^{\prime} \cap \cdots \cap D_{i}^{\prime} \cap \cdots \cap D_{n}^{\prime} \quad \text { and } \quad S_{2}=D_{1}^{\prime} \cap \cdots \cap D_{i}^{\prime \prime} \cap \cdots \cap D_{n}^{\prime} .
$$

Since the number $\left[f_{0}, \ldots, f_{n}\right]_{x}^{c(x)}$ is multiplicatively skew-symmetric in $D_{1}, \ldots, D_{n}$ (see Remark 3.4) we may assume that $i=1$, so

$$
S_{1}=D_{1}^{\prime} \cap D_{2}^{\prime} \cdots \cap D_{n}^{\prime} \quad \text { and } \quad S_{2}=D_{1}^{\prime \prime} \cap D_{2}^{\prime} \cdots \cap D_{n}^{\prime}
$$

We have to show that

$$
\prod_{x \in S_{1} \cup S_{2}}\left[f_{0}, \ldots, f_{n}\right]_{x}^{c(x)}=1 .
$$

Let $\Sigma$ be the union of all stratum closures $Y \in \overline{\mathrm{St}}_{1}(X)$ with signature $\left\{2^{\prime}, \ldots, n^{\prime}\right\}$. It follows from Corollary 3.13 that if $x \in S_{1} \cup S_{2}$ does not lie on any component of $\Sigma$ then $\left[f_{0}, \ldots, f_{n}\right]_{x}^{c(x)}=1$. On the other hand, by (5) the signature of every point $x \in \operatorname{St}_{0}(X) \cap \Sigma$ is either

$$
\left\{2^{\prime}, \ldots, n^{\prime}\right\} \quad \text { or } \quad\left\{1^{\prime}, 2^{\prime}, \ldots, n^{\prime}\right\} \quad \text { or } \quad\left\{1^{\prime \prime}, 2^{\prime}, \ldots, n^{\prime}\right\} .
$$




\section{SOPROUNOV}

In the first case $\left[f_{0}, \ldots, f_{n}\right]_{x}^{c(x)}=1$, again by Corollary 3.13. In the second case $x \in S_{1}$; and in the third $x \in S_{2}$. Therefore, we have

$$
\prod_{x \in S_{1} \cup S_{2}}\left[f_{0}, \ldots, f_{n}\right]_{x}^{c(x)}=\prod_{x \in \operatorname{St}_{0}(X) \cap \Sigma}\left[f_{0}, \ldots, f_{n}\right]_{x}^{c(x)} .
$$

Now consider a component $Y$ of $\Sigma$, and a closed point $y \in Y$. Let $\mathcal{F}(y, Y)$ denote the set of all complete flags

$$
y \subset Y \subset X_{2} \subset \cdots \subset X_{n-1} \subset X
$$

where $X_{i} \in \overline{\operatorname{St}}_{i}(X)$ has signature $\left\{(i+1)^{\prime}, \ldots, n^{\prime}\right\}, 2 \leqslant i \leqslant n-1$. Denote

$$
\left\langle f_{0}, \ldots, f_{n}\right\rangle_{y, Y}=\prod_{F \in \mathcal{F}(y, Y)}\left\langle f_{0}, \ldots, f_{n}\right\rangle_{F},
$$

and assume that $\left\langle f_{0}, \ldots, f_{n}\right\rangle_{y, Y}=1$ if $\mathcal{F}(y, Y)$ is empty. Then by Corollary 3.13 for each $x \in$ $\mathrm{St}_{0}(X) \cap \Sigma$ we have

$$
\left[f_{0}, \ldots, f_{n}\right]_{x}^{c(x)}=\prod_{F \in \mathcal{F}(x)}\left\langle f_{0}, \ldots, f_{n}\right\rangle_{F}=\prod_{Y \ni x}\left\langle f_{0}, \ldots, f_{n}\right\rangle_{x, Y},
$$

where the product on the right-hand side runs over all components $Y$ of $\Sigma$ containing $x$.

On the other hand, by the first Parshin's reciprocity law (Theorem A.4 for $i=0$ )

$$
\prod_{y \in Y}\left\langle f_{0}, \ldots, f_{n}\right\rangle_{y \subset Y \subset X_{2} \subset \cdots \subset X_{n-1} \subset X}=1,
$$

where the product is taken over all points $y \in Y$. Thus

$$
\prod_{y \in Y}\left\langle f_{0}, \ldots, f_{n}\right\rangle_{y, Y}=1
$$

Note that $\left\langle f_{0}, \ldots, f_{n}\right\rangle_{y, Y}$ is trivial for all points $y$ not lying in $\operatorname{St}_{0}(X)$, so we can assume that $y \in \operatorname{St}_{0}(X) \cap Y$. We have

$$
\prod_{y \in \operatorname{St}_{0}(X) \cap Y}\left\langle f_{0}, \ldots, f_{n}\right\rangle_{y, Y}=1
$$

Combining (7), (8) and (9) we get

$$
\begin{aligned}
\prod_{x \in S_{1} \cup S_{2}}\left[f_{0}, \ldots, f_{n}\right]_{x}^{c(x)} & =\prod_{x \in \operatorname{St}_{0}(X) \cap \Sigma}\left[f_{0}, \ldots, f_{n}\right]_{x}^{c(x)} \\
& =\prod_{x \in \operatorname{St}_{0}(X) \cap \Sigma} \prod_{Y \ni x}\left\langle f_{0}, \ldots, f_{n}\right\rangle_{x, Y} \\
& =\prod_{Y \subset \Sigma} \prod_{x \in \operatorname{St}_{0}(X) \cap Y}\left\langle f_{0}, \ldots, f_{n}\right\rangle_{x, Y}=1 .
\end{aligned}
$$

\section{Toroidal residue}

Let $(X, D)$ be a toroidal pair, as before. At each point $x \in \operatorname{St}_{0}(X)$ we define the residue $\operatorname{res}_{x}^{\mathbb{T}} \omega$ of a rational $n$-form $\omega$ on $X$ which is regular in $X \backslash D$. Then we prove an additive analog of Theorem 3.15.

\subsection{Toroidal residue}

First we will define the toroidal residue for a local model $\left(X_{\sigma}, x_{0}, \omega_{0}\right)$ at a point $x \in \mathrm{St}_{0}(X)$. As before $X_{\sigma}$ is an $n$-dimensional affine toric variety, $x_{0}$ is the closed orbit, and $\omega_{0}=d t_{1} / t_{1} \wedge \cdots \wedge d t_{n} / t_{n}$, where $\left(t_{1}, \ldots, t_{n}\right)$ is a coordinate system in $\mathbb{T}$. 


\section{RESIDUES AND TAME SYMBOLS ON TOROIDAL VARIETIES}

Let $A=\hat{\mathcal{O}}_{X_{\sigma}, x_{0}}$ be the completion of the local ring of $x_{0}$ on $X_{\sigma}$, and $B=A_{S}$ the localization of $A$ by the multiplicative subgroup $S$ of all monomials. We consider the $B$-algebra $\Omega_{B}^{n}$ of differential $n$-forms that are regular in $\mathbb{T}$, and the $A$-algebra $\Omega_{A}^{n}$ of regular differential $n$-forms.

By fixing coordinates $\left(t_{1}, \ldots, t_{n}\right)$ we can identify every element $f \in B$ with a formal power series

$$
f=t^{b} \sum_{a \in \sigma \cap \mathbb{Z}^{n}} \lambda_{a} t^{a}, \quad b \in \mathbb{Z}^{n} .
$$

Let $\omega \in \Omega_{B}^{n}$. Then we can write $\omega=f d t_{1} / t_{1} \wedge \cdots \wedge d t_{n} / t_{n}$, for some $f \in B$.

Definition 4.1. The toroidal residue of a differential $n$-form $\omega \in \Omega_{B}^{n}$ is the constant term $\lambda_{-b}$ in the formal power series of $f$. We denote it by $\operatorname{res}^{\mathbb{T}} \omega$.

We have the following properties of the toroidal residue.

Proposition 4.2. Consider a differential $n$-form $\omega \in \Omega_{B}^{n}$. Then the following hold.

i) If $\omega$ is exact then $\operatorname{res}^{\mathbb{T}} \omega=0$.

ii) If $\omega \in \Omega_{A}^{n}$ then $\operatorname{res}^{\mathbb{T}} \omega=0$.

iii) For any $s_{1}, \ldots, s_{n} \in B^{\times}, \operatorname{res}^{\mathbb{T}} d s_{1} / s_{1}^{m_{1}} \wedge \cdots \wedge d s_{n} / s_{n}^{m_{n}}=0$, unless all $m_{i}=1$.

iv) The toroidal residue is independent of the choice of coordinates $\left(t_{1}, \ldots, t_{n}\right)$.

v) The toroidal residue is invariant under monomial transformations $t \mapsto t^{Q}, Q \in G L(n, \mathbb{Z})$, up to a factor $\operatorname{det} Q$.

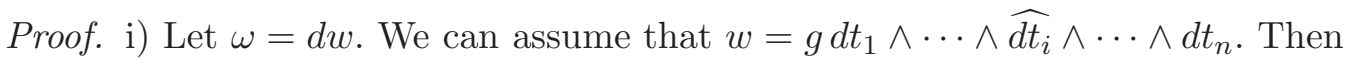

$$
\omega=(-1)^{i-1} \frac{\partial g}{\partial t_{i}} t_{1} \ldots t_{n} \frac{d t_{1}}{t_{1}} \wedge \cdots \wedge \frac{d t_{n}}{t_{n}} .
$$

Suppose $g=\sum_{a} \lambda_{a} t^{a}$. Then

$$
(-1)^{i-1} \frac{\partial g}{\partial t_{i}} t_{1} \ldots t_{n}=\sum_{a} a_{i} \lambda_{a} \frac{t^{a}}{t_{i}} t_{1} \ldots t_{n}, \quad a=\left(a_{1}, \ldots, a_{n}\right)
$$

Clearly the constant term of the last series is zero.

ii) Let $u_{i}=t^{a_{i}}, a_{i} \in \sigma \cap \mathbb{Z}^{n}$, be $n$ regular functions, such that $d u_{1}, \ldots, d u_{n}$ are linearly independent. Then for every $\omega \in \Omega_{A}^{n}$

$$
\omega=f d u_{1} \wedge \cdots \wedge d u_{n}=f d t^{a_{1}} \wedge \cdots \wedge d t^{a_{n}}=f t^{a_{1}+\cdots+a_{n}} J \frac{d t_{1}}{t_{1}} \wedge \cdots \wedge \frac{d t_{n}}{t_{n}},
$$

where $f \in A$ and $J=\operatorname{det}\left(a_{1}, \ldots, a_{n}\right)$. Clearly, the constant term of $f t^{a_{1}+\cdots+a_{n}} J$ is zero.

iii) First assume that $\operatorname{char}(k)=0$. Suppose $m_{i} \neq 1$. Then

$$
\frac{d s_{1}}{s_{1}^{m_{1}}} \wedge \cdots \wedge \frac{d s_{n}}{s_{n}^{m_{n}}}=d\left(\frac{(-1)^{i-1}}{1-m_{i}} s_{i}^{1-m_{i}} \frac{d s_{1}}{s_{1}^{m_{1}}} \wedge \cdots \wedge \frac{\widehat{d s_{i}}}{s_{i}^{m_{i}}} \wedge \cdots \wedge \frac{d s_{n}}{s_{n}^{m_{n}}}\right),
$$

and the statement follows from part i. In the case of an arbitrary characteristic, note that the toroidal residue is a polynomial function in finitely many coefficients of the series $s_{1}, \ldots, s_{n} \in B^{\times}$. This function is independent of the characteristic and vanishes when the characteristic is zero. Therefore it is identically zero.

iv) Let $\left(s_{1}, \ldots, s_{n}\right)$ be another coordinate system in $\mathbb{T}$. Then $s_{i}=\phi_{i} t_{i}$, where $\phi_{i} \in A^{\times}$. Consider $\omega \in \Omega_{B}^{n}$ and write

$$
\omega=f \frac{d s_{1}}{s_{1}} \wedge \cdots \wedge \frac{d s_{n}}{s_{n}}, \quad f=\sum_{a} \lambda_{a} s^{a}, \quad a \in \mathbb{Z}^{n}
$$




\section{Soprounov}

Then the residue of $\omega$ with respect to $\left(s_{1}, \ldots, s_{n}\right)$ is $\operatorname{res}_{\left(s_{1}, \ldots, s_{n}\right)}^{\mathbb{T}} \omega=\lambda_{0}$. On the other hand, by part iii the residue of $\omega$ with respect to $\left(t_{1}, \ldots, t_{n}\right)$ equals

$$
\operatorname{res}_{\left(t_{1}, \ldots, t_{n}\right)}^{\mathbb{T}} \omega=\operatorname{res}_{\left(t_{1}, \ldots, t_{n}\right)}^{\mathbb{T}} \lambda_{0} \frac{d s_{1}}{s_{1}} \wedge \cdots \wedge \frac{d s_{n}}{s_{n}} .
$$

Now, for each $1 \leqslant i \leqslant n$,

$$
\frac{d s_{i}}{s_{i}}=\frac{d \phi_{i}}{\phi_{i}}+\frac{d t_{i}}{t_{i}}
$$

Substituting into (10) and expanding we get

$$
\operatorname{res}_{\left(t_{1}, \ldots, t_{n}\right)}^{\mathbb{T}} \omega=\operatorname{res}_{\left(t_{1}, \ldots, t_{n}\right)}^{\mathbb{T}} \lambda_{0} \frac{d t_{1}}{t_{1}} \wedge \cdots \wedge \frac{d t_{n}}{t_{n}}+\sum_{i} \operatorname{res}_{\left(t_{1}, \ldots, t_{n}\right)}^{\mathbb{T}} \omega_{i},
$$

where in each $\omega_{i}$ at least one of the $d t_{j} / t_{j}$ is replaced by $d \phi_{j} / \phi_{j}$. It is easy to check that the residue of every $\omega_{i}$ is zero.

v) This follows from the fact that if $u_{i}=t^{q_{i}}, q_{i} \in \mathbb{Z}^{n}$, then

$$
\frac{d u_{1}}{u_{1}} \wedge \cdots \wedge \frac{d u_{n}}{u_{n}}=\operatorname{det} Q \frac{d t_{1}}{t_{1}} \wedge \cdots \wedge \frac{d t_{n}}{t_{n}},
$$

and from the observation that monomial transformations do not change the constant term of a series.

Remark 4.3. The proof of parts iii and iv is similar to the one given in [FP04] for Parshin's residue.

Now let $(X, D)$ be toroidal. Denote by $\Omega^{n}(X, D)$ the set of all rational $n$-forms on $X$ that are regular in $X \backslash D$.

Definition 4.4. The toroidal residue of a rational $n$-form $\omega \in \Omega^{n}(X, D)$ at a point $x \in \operatorname{St}_{0}(X)$ is the toroidal residue of its image in a local model at $x$. We denote it by $\operatorname{res}_{x}^{\mathbb{T}} \omega$.

Remark 4.5 (Invariance). As follows from Propositions 3.1 and 4.2, the toroidal residue is the same for any two equipped local models that are related by an isomorphism preserving the form $\omega_{0}$, and changes sign otherwise.

The relation between the toroidal residue and Parshin's residue is similar to the one between the toroidal symbol and Parshin's tame symbol.

Proposition 4.6. Let $(X, D)$ be toroidal. Consider a rational $n$-form $\omega \in \Omega^{n}(X, D)$. Then for any complete flag $F: X_{0} \subset X_{1} \subset \cdots \subset X_{n-1} \subset X$ of stratum closures on $X$ we have

$$
\operatorname{res}_{F} \omega=\operatorname{sgn} F \operatorname{res}_{X_{0}}^{\mathbb{T}} \omega
$$

where $\operatorname{res}_{F} \omega$ denotes Parshin's residue at the flag $F$.

The number $\operatorname{sgn} F$ res $^{\mathbb{T}} \omega$ is independent of the choice of the form $\omega_{0}$ in an equipped local model. Consequently, if $\left(D_{1}, \ldots, D_{n}\right)$ is a reasonable covering of $D$ then at each $x \in \operatorname{St}_{0}(X)$ the number $c(x) \operatorname{res}_{x}^{\mathbb{T}} \omega$ is also well defined.

The following is the additive analog of Corollary 3.13.

Corollary 4.7. Let $(X, D)$ be toroidal and $\left(D_{1}, \ldots, D_{n}\right)$ be a reasonable covering of $D$. For $x \in \mathrm{St}_{0}(X)$ let $\mathcal{F}(x)$ be the set of all complete flags

$$
x=X_{0} \subset X_{1} \subset \cdots \subset X_{n-1} \subset X,
$$

where $X_{i} \subset \overline{\operatorname{St}}_{i}(X)$ is a stratum closure of signature $\{i+1, \ldots, n\}, 0 \leqslant i \leqslant n-1$. 


\section{RESIDUES AND TAME SYMBOLS ON TOROIDAL VARIETIES}

Then for any $\omega \in \Omega^{n}(X, D)$ we have

$$
c(x) \operatorname{res}_{x}^{\mathbb{T}} \omega=\sum_{F \in \mathcal{F}(x)} \operatorname{res}_{F} \omega,
$$

where we assume that the sum is 0 if $\mathcal{F}(x)$ is empty.

\subsection{Main theorem}

TheOREM 4.8. Let $X$ be a complete normal $n$-dimensional variety over an algebraically closed field $k$, and $D$ a closed subset of $X$ such that the pair $(X, D)$ is toroidal.

Let $\left(D_{1}, \ldots, D_{n}\right)$ be a reasonable covering of $D$ such that each $D_{i}$ is a disjoint union of two closed subsets of pure codimension 1:

$$
D=D_{1} \cup \cdots \cup D_{n}, \quad D_{1} \cap \cdots \cap D_{n} \subset \operatorname{St}_{0}(X), \quad D_{i}=D_{i}^{\prime} \sqcup D_{i}^{\prime \prime}, \quad 1 \leqslant i \leqslant n .
$$

We get $2^{n}$ disjoint finite closed subsets of $X$ :

$$
S_{k}=G_{1} \cap \cdots \cap G_{n}, \text { where } G_{i}=D_{i}^{\prime} \text { or } D_{i}^{\prime \prime}, \quad 1 \leqslant i \leqslant n, \quad 1 \leqslant k \leqslant 2^{n} .
$$

Then for any rational $n$-form $\omega \in \Omega^{n}(X, D)$, the following $2^{n}$ numbers are equal:

$$
(-1)^{\left|S_{1}\right|} \sum_{x \in S_{1}} c(x) \operatorname{res}_{x}^{\mathbb{T}} \omega=\cdots=(-1)^{\left|S_{2^{n}}\right|} \sum_{x \in S_{2^{n}}} c(x) \operatorname{res}_{x}^{\mathbb{T}} \omega,
$$

where $\operatorname{res}_{x}^{\mathbb{T}} \omega$ denotes the toroidal residue of $\omega$ at $x, c(x)$ is the combinatorial coefficient at $x$, and $\left|S_{k}\right|$ is the number of $D_{i}^{\prime \prime}$ in the definition of $S_{k}$.

Proof. The proof repeats the arguments of the proof of Theorem 3.15.

\section{Application to systems of equations in the torus}

In this section we apply our main results on the toroidal symbol and residue to prove the product of roots formula and the sum of values formula (Theorems A and B in the Introduction).

Recall that a Laurent polynomial is a finite linear combination of monomials with integer exponent vectors and coefficients in $k$ :

$$
f(t)=\sum_{m \in \mathbb{Z}^{n}} \lambda_{m} t^{m}, \quad t^{m}=t_{1}^{m_{1}} \ldots t_{n}^{m_{n}}, \quad \lambda_{m} \in k .
$$

The convex hull of those lattice points $m \in \mathbb{Z}^{n}$ for which $\lambda_{m} \neq 0$ is called the Newton polytope of $f$. The value of $f(t)$ is defined for all $t$ in the algebraic $n$-torus $\mathbb{T}=\left(k^{\times}\right)^{n}$.

Consider a system of $n$ Laurent polynomial equations in $\mathbb{T}$ :

$$
f_{1}(t)=\cdots=f_{n}(t)=0, \quad t \in \mathbb{T} .
$$

Let $\Delta_{i}$ be the Newton polytope of $f_{i}$. We assume that none of the $f_{i}$ is a monomial, hence, none of $\Delta_{i}$ is a point.

Every linear functional $w$ on $\mathbb{R}^{n}$ defines a collection of faces $\Delta_{1}^{w}, \ldots, \Delta_{n}^{w}$ of the Newton polytopes such that the restriction of $w$ on $\Delta_{i}$ attains its maximum precisely at $\Delta_{i}^{w}$. The polynomial

$$
f_{i}^{w}(t)=\sum_{m \in \Delta_{i}^{w} \cap \mathbb{Z}^{n}} \lambda_{m} t^{m}
$$

is called the initial form of $f_{i}$ with respect to $w$. According to Bernstein's theorem [Ber75] the number of solutions to the system (12) is finite (and equals the mixed volume of $\Delta_{1}, \ldots, \Delta_{n}$ ) if and only if for every $w \neq 0$ the system $f_{1}^{w}(t)=\cdots=f_{n}^{w}(t)=0$ is inconsistent. 


\section{Soprounov}

Definition 5.1. A collection of polytopes $\Delta_{1}, \ldots, \Delta_{n}$ is called developed if none of them is a point and for each $w \neq 0$ at least one of the faces $\Delta_{1}^{w}, \ldots, \Delta_{n}^{w}$ is a vertex.

By the above any system with developed collection of Newton polytopes has finitely many solutions. We will call them the roots of the system. Every root $x$ of the system has a multiplicity $\mu(x)$.

Let $\Delta$ be the Minkowski sum of $\Delta_{1}, \ldots, \Delta_{n}$. Then every face $\Gamma \subset \Delta$ has a unique decomposition as a sum of faces

$$
\Gamma=\Gamma_{1}+\cdots+\Gamma_{n}, \quad \text { where } \quad \Gamma_{i} \subset \Delta_{i}, \quad i=1, \ldots, n .
$$

If the collection $\Delta_{1}, \ldots, \Delta_{n}$ is developed then $\Delta$ has dimension $n$ and in the decomposition of every proper face of $\Delta$ at least one summand is a vertex. In this case, for each vertex $A$ of $\Delta$, the combinatorial coefficient $c(A)$ is defined.

Definition 5.2. Let $\sigma_{A}$ be the cone with apex $A$ generated by the facets of $\Delta$ that contain $A$. Then the boundary of $\sigma_{A}$ is covered by the closed sets $D_{1}, \ldots, D_{n}$, where $D_{i}$ is the union of all facets of $\sigma_{A}$ that correspond to the facets of $\Delta$ whose $i$ th summand in the decomposition (13) is a vertex. The combinatorial coefficient of this covering is called the combinatorial coefficient $c(A)$ of the vertex $A \in \Delta$.

\subsection{Product of roots formula}

Consider a system of $n$ Laurent polynomials with developed collection of Newton polytopes. The product of the roots counting multiplicities is a point in $\mathbb{T}$, which we denote by $\rho$. To locate $\rho$ it is enough to find the product of the values of $t_{i}$ over the roots of the system, for each $1 \leqslant i \leqslant n$. More generally, we will find the product of the values of any Laurent monomial $c t^{m}$, for $c \in k^{\times}, m \in \mathbb{Z}^{n}$, over the roots of the system.

Definition 5.3 [Kho99]. The symbol of $f_{1}, \ldots, f_{n}$ and a Laurent monomial $f_{0}=c^{m}$ at a vertex $A \in \Delta$ is the symbol of $n+1$ monomials $\left[c t^{m}, f_{1}\left(A_{1}\right) t^{A_{1}}, \ldots, f_{n}\left(A_{n}\right) t^{A_{n}}\right]$, where $A=A_{1}+\cdots+A_{n}$ is the decomposition of $A$, and $f_{i}\left(A_{i}\right)$ is the coefficient of $t^{A_{i}}$ in $f_{i}$. We denote it by $\left[f_{0}, \ldots, f_{n}\right]_{A}$.

The following theorem was proved by Khovanskii [Kho99] in the complex case. Our proof uses the result of Theorem 3.15 and works over an arbitrary algebraically closed field $k$.

Theorem 5.4. Suppose the collection of the Newton polytopes $\Delta_{1}, \ldots, \Delta_{n}$ of the system (12) is developed. Then the product of the values of a Laurent monomial $f_{0}$ over the roots of the system is given by

$$
\prod_{f_{1}(x)=\cdots=f_{n}(x)=0} f_{0}(x)^{\mu(x)}=\prod_{A}\left[f_{0}, \ldots, f_{n}\right]_{A}^{(-1)^{n} c(A)},
$$

where the right-hand product is taken over all vertices $A$ of the polytope $\Delta=\Delta_{1}+\cdots+\Delta_{n}$ and $c(A)$ is the combinatorial coefficient at $A$.

Proof. First let us notice that it is sufficient to prove the theorem for a generic system with given Newton polytopes. There is an open set $U$ in the space of the coefficients of the system where the number of roots counting multiplicities is constant. The left-hand side of (14), being symmetric in the roots of the system, is a rational function in the coefficients of the system. On the other hand, the product of the symbols $\left[f_{0}, \ldots, f_{n}\right]_{A}$ is also a rational function of the coefficients of the system. Suppose we proved the formula for almost all systems in $U$. Then the two rational functions coincide on an open algebraic subset $W \subset U$, and thus coincide everywhere in $U$.

Consider the complete toric variety $X$ associated with the Minkowski sum $\Delta$ (e.g. see [Ful93]). Let $D=X \backslash \mathbb{T}$ be the invariant divisor on $X$. Denote by $Z_{i}$ the closure of the zero locus $f_{i}=0$ in $X$, 


\section{RESIDUES AND TAME SYMBOLS ON TOROIDAL VARIETIES}

and let $Z=Z_{1} \cup \cdots \cup Z_{n}$. If the system is generic, the components of $Z$ intersect transversally and the intersection of each component of $Z$ with $D$ is also transversal. In this case the pair $(X, D \cup Z)$ is toroidal.

Now we will define a covering of $D \cup Z$. Each irreducible component of $D$ corresponds to a facet of $\Delta$. Recall that each facet $\Gamma$ of $\Delta$ has a unique decomposition into the sum of faces (13). Denote by $D_{i}$ the union of all components that correspond to those facets whose $i$ th summand is a vertex. Since the collection $\Delta_{1}, \ldots, \Delta_{n}$ is developed, the sets $D_{1}, \ldots, D_{n}$ define a covering of $D$.

Consider a covering $D \cup Z=\left(D_{1} \cup Z_{1}\right) \cup \cdots \cup\left(D_{n} \cup Z_{n}\right)$. Notice that every intersection point in $\left(D_{1} \cup Z_{1}\right) \cap \cdots \cap\left(D_{n} \cup Z_{n}\right)$ is either a fixed orbit or a transversal intersection of some components of $Z$ and $D$. Thus the covering is reasonable (see $\S 3.2$ ). By definition, the components of $D_{i}$ correspond to facets of $\Delta$ whose $i$ th summand is a vertex, i.e. the corresponding initial forms of $f_{i}$ are monomials. This implies that $D_{i} \cap Z_{i}=\emptyset$. Now applying Theorem 3.15 we obtain

$$
\prod_{x \in Z_{1} \cap \cdots \cap Z_{n}}\left[f_{0}, \ldots, f_{n}\right]_{x}^{c(x)}=\prod_{x \in D_{1} \cap \cdots \cap D_{n}}\left[f_{0}, \ldots, f_{n}\right]_{x}^{(-1)^{n} c(x)} .
$$

It remains to notice that for each transversal intersection $x$ of components of $Z$ the combinatorial coefficient $c(x)=1$ and $\left[f_{0}, \ldots, f_{n}\right]_{x}=f_{0}(x)^{\mu(x)}$ (see Example 1 in the Appendix). Also for a point $x \in D_{1} \cap \cdots \cap D_{n}$ the toroidal symbol $\left[f_{0}, \ldots, f_{n}\right]_{x}$ coincides with $\left[f_{0}, \ldots, f_{n}\right]_{A}$, where $A$ is the corresponding vertex of $\Delta$, and $c(x)=c(A)$, by definition.

\subsection{Sum of values formula}

We recall the definition of a Laurent series at a vertex and the residue at a vertex from [GK96]. Let $f$ be a Laurent polynomial with Newton polytope $\Delta(f), A$ a vertex of $\Delta(f)$, and $f(A)$ the coefficient of $t^{A}$ in $f$. Since the constant term of the Laurent polynomial $\tilde{f}=f /\left(f(A) t^{A}\right)$ equals 1 , we get a well-defined power series

$$
\frac{1}{\tilde{f}}=1+(1-\tilde{f})+(1-\tilde{f})^{2}+\cdots .
$$

Definition 5.5. Let $g$ be a Laurent polynomial. The formal product of the series (15) and the Laurent polynomial $g /\left(f(A) t^{A}\right)$ is called the Laurent series of $g / f$ at the vertex $A \in \Delta(f)$.

Definition 5.6. The residue at a vertex $A \in \Delta(f)$ of a rational $n$-form

$$
\omega=\frac{g}{f} \frac{d t_{1}}{t_{1}} \wedge \cdots \wedge \frac{d t_{n}}{t_{n}}
$$

is the constant term of the Laurent series of $g / f$ at $A$. We denote it by $\operatorname{res}_{A} \omega$.

The following theorem was proved by Gel'fond and Khovanskii [GK02] in the case when $k=\mathbb{C}$. We prove it for any algebraically closed field $k$ using Theorem 4.8.

Theorem 5.7. Suppose the collection of the Newton polytopes $\Delta_{1}, \ldots, \Delta_{n}$ of the system (12) is developed. Then the sum of the values of a Laurent polynomial $f_{0}$ over the roots of the system counting multiplicities is given by

$$
\sum_{f_{1}(x)=\cdots=f_{n}(x)=0} \mu(x) f_{0}(x)=(-1)^{n} \sum_{A} c(A) \operatorname{res}_{A}\left(\frac{f_{0} J}{f} \frac{d t_{1}}{t_{1}} \wedge \cdots \wedge \frac{d t_{n}}{t_{n}}\right),
$$

where the sum on the right is taken over the vertices $A$ of $\Delta=\Delta_{1}+\cdots+\Delta_{n}, J=\operatorname{det}\left(t_{j} \partial f_{i} / \partial t_{j}\right)$ for $1 \leqslant i, j \leqslant n, f=f_{1} \cdots f_{n}$, and $c(A)$ is the combinatorial coefficient at $A$.

Proof. As in the proof of Theorem 5.4 it is enough to consider the case of a generic system with given Newton polytopes, since the sum of the values of a Laurent polynomial over the roots of the system is a rational function of the coefficients of the system. 


\section{Soprounov}

As before, $X$ is the complete toric variety associated with the Minkowski sum, $D$ the invariant divisor on $X, Z_{i}$ the closure of the zero locus $f_{i}=0$ in $X$, and $D \cup Z=\left(D_{1} \cup Z_{1}\right) \cup \cdots \cup\left(D_{n} \cup Z_{n}\right)$ a reasonable covering that satisfies $D_{i} \cap Z_{i}=\emptyset$. Applying Theorem 4.8 to the form

$$
\omega=f_{0} \frac{d f_{1}}{f_{1}} \wedge \cdots \wedge \frac{d f_{n}}{f_{n}}
$$

we get

$$
\sum_{x \in Z_{1} \cap \cdots \cap Z_{n}} c(x) \operatorname{res}_{x}^{\mathbb{T}} \omega=(-1)^{n} \sum_{x \in D_{1} \cap \cdots \cap D_{n}} c(x) \operatorname{res}_{x}^{\mathbb{T}} \omega .
$$

For each transversal intersection $x$ of components of $Z$ the combinatorial coefficient $c(x)=1$ and $\operatorname{res}_{x}^{\mathbb{T}} \omega=\mu(x) f_{0}(x)$ (see Example 2 in the Appendix). Also for a point $x \in D_{1} \cap \cdots \cap D_{n}$, we have $c(x)=c(A)$ and

$$
\operatorname{res}_{x}^{\mathbb{T}} \omega=\operatorname{res}_{A}\left(\frac{f_{0} J}{f} \frac{d t_{1}}{t_{1}} \wedge \cdots \wedge \frac{d t_{n}}{t_{n}}\right)
$$

where $A \in \Delta$ is the vertex corresponding to the fixed orbit $x$. Therefore, we have obtained the required equality.

\section{ACKNOWLEDGEMENTS}

The results of the present paper constitute a part of the author's PhD thesis [Sop02]. I am grateful to Askold Khovanskii for stating the problem, his constant support and numerous discussions.

\section{Appendix. Parshin's reciprocity laws}

Here we recall the definition of Parshin's tame symbol and residue for an arbitrary algebraic variety $X$ over an algebraically closed field, and formulate Parshin's reciprocity laws.

\section{A.1 Parshin's tame symbol}

Let $X$ be a complete algebraic variety over an algebraically closed field $k$.

Consider a complete flag of irreducible subvarieties of $X$ :

$$
F: X_{0} \subset X_{1} \subset \cdots \subset X_{n-1} \subset X_{n}=X .
$$

We will assume that all $X_{i}$ are normal. The general case can be reduced to this one by considering normalization (for details see [Par83] or [Sop02]). Note also that this assumption holds for complete flags of stratum closures on toroidal pairs.

Given a flag $F$ as in (A1) define a system of local parameters at $F,\left(u_{1}, \ldots, u_{n}\right)_{F} \in k(X)^{n}$, as follows. There exists an open subset of $X_{n}$ where the codimension- 1 subvariety $X_{n-1}$ has a local equation $u_{n}$. In general, for every $i=0, \ldots, n-1$, let $u_{n-i}$ be a local equation (in some open subset) of the codimension- 1 subvariety $X_{n-i-1} \subset X_{n-i}$.

Next, for every rational function $f$ on $X$ define its order $\left(a_{1}, \ldots, a_{n}\right)_{F} \in \mathbb{Z}^{n}$ at the flag $F$. First let $a_{n}$ be the order of $f$ along $X_{n-1}$. We can write

$$
f=f^{(n-1)} u_{n}^{a_{n}}, \quad a_{n} \in \mathbb{Z}
$$

Let $\tilde{f}^{(n-1)}$ be the restriction of $f^{(n-1)}$ to $X_{n-1}$, and $a_{n-1}$ be the order of this restriction along $X_{n-2}$,

$$
\tilde{f}^{(n-1)}=f^{(n-2)} u_{n-1}^{a_{n-1}}, \quad a_{n-1} \in \mathbb{Z},
$$

and so on. Finally,

where $a_{1}$ is the order of $\tilde{f}^{(1)}$ at $X_{0}$.

$$
\tilde{f}^{(1)}=f^{(0)} u_{1}^{a_{1}}, \quad a_{1} \in \mathbb{Z},
$$




\section{RESIDUES AND TAME SYMBOLS ON TOROIDAL VARIETIES}

Definition A.1. Let $f_{0}, \ldots, f_{n}$ be rational functions on $X$. Fix a complete flag $F$ of irreducible subvarieties (A1). Let $\left(a_{i 1}, \ldots, a_{i n}\right)_{F}$ be the order of $f_{i}$ at $F$. Denote $A=\left(a_{i j}\right) \in M_{n+1, n}(\mathbb{Z})$. Parshin's tame symbol of $f_{0}, \ldots, f_{n}$ at the flag $F$ is the following non-zero element of $k$ :

$$
\left\langle f_{0}, \ldots, f_{n}\right\rangle_{F}=(-1)^{B}\left(\prod_{i=0}^{n} f_{i}^{(-1)^{i} A_{i}}\right)\left(X_{0}\right),
$$

where $A_{i}$ is the determinant of the matrix obtained from $A$ by eliminating its $i$ th row, and

$$
B=\sum_{k} \sum_{i<j} a_{i k} a_{j k} A_{i j}^{k}
$$

where $A_{i j}^{k}$ is the determinant of the matrix obtained from $A$ by eliminating its $i$ th and $j$ th rows and its $k$ th column.

Note that the order of the rational function inside the large brackets in $(\mathrm{A} 3)$ is $(0, \ldots, 0)_{F}$, hence, its value at $X_{0}$ makes sense and is not zero.

Remark A.2. Let us associate with every rational function $f$ on $X$ a monomial $c u_{1}^{a_{1}} \ldots u_{n}^{a_{n}}$, where $\left(u_{1}, \ldots, u_{n}\right)_{F}$ are local parameters at $F,\left(a_{1}, \ldots, a_{n}\right)_{F}$ is the order of $f$ at $F$, and $c=f^{(0)}\left(X_{0}\right)$. Then the tame symbol $\left\langle f_{0}, \ldots, f_{n}\right\rangle_{F}$ is equal to the symbol of the corresponding $n+1$ monomials (see Definition 3.8).

Parshin's symbol does not depend on the choice of local parameters $\left(u_{1}, \ldots, u_{n}\right)_{F}$. It is multiplicative and skew-symmetric (compare to Proposition 3.9).

Example 1. Let $f_{1}, \ldots, f_{n}$ be rational functions on an algebraic variety $X$, whose zero loci $\left\{f_{i}=0\right\}$ intersect transversely at a non-singular point $x \in X$. Denote by $Z_{i}$ the irreducible component of $\left\{f_{i}=0\right\}$ that contains $x$.

Let $f_{0}$ be any rational function on $X$ whose divisor does not contain $x$. Then

$$
\left\langle f_{0}, \ldots, f_{n}\right\rangle_{F}=f_{0}(x)^{\mu(x)},
$$

where $F: x=X_{0} \subset X_{1} \subset \cdots \subset X_{n-1} \subset X$ for $X_{i}=Z_{i+1} \cap \cdots \cap Z_{n}$, and $\mu(x)=\mu_{1} \ldots \mu_{n}$ is the product of the multiplicities $\mu_{i}$ of $f_{i}$ along $Z_{i}$.

Indeed, for the system of local parameters at $F$ we can choose the local equations of $Z_{i}$ at $x$. Then the first row of the matrix $A$ is zero and the other $n$ rows form a lower triangular matrix with the multiplicities $\mu_{i}$ on the diagonal. Therefore, $A_{0}=\mu_{1} \ldots \mu_{n}$ and $A_{j}=0,1 \leqslant j \leqslant n$. It is also not hard to see that $B=0$ (for example, one can use the description of $B$ given in the proof of Proposition 3.9).

\section{A.2 Parshin's residue}

Let $X$ be a complete algebraic variety over an algebraically closed field $k$, and $F$ a complete flag of irreducible subvarieties (A1). Let $\left(u_{1}, \ldots, u_{n}\right)_{F}$ be a system of local parameters at $F$, as before.

Consider a rational differential $n$-form on $X$. At a generic point of $X_{n-1}$ the differentials $d u_{1}, \ldots, d u_{n}$ are linearly independent, and we can write

$$
\omega=f d u_{1} \wedge \cdots \wedge d u_{n}, \quad \text { where } f=\sum_{i_{n}>N_{n}} f_{i_{n}} u_{n}^{i_{n}} .
$$

The restriction of the form $f_{-1} d u_{1} \wedge \cdots \wedge u_{n-1}$ onto $X_{n-1}$ makes sense and gives us a rational $(n-1)$-form $\omega_{n-1}$ on $X_{n-1}$. Continuing in this way we arrive at a sequence of rational $(n-i)$-forms $\omega_{n-i}$ on $X_{n-i}, i=1, \ldots, n$, the last one being a number $\omega_{0}=f_{-1, \ldots,-1}$ at the point $X_{0}$. Note also 


\section{Soprounov}

that this number is the coefficient of the series

$$
f=\sum_{i_{n} \geqslant N_{n}} \sum_{i_{n-1} \geqslant N_{n-1}\left(i_{n}\right)} \ldots \sum_{i_{1} \geqslant N_{1}\left(i_{2}, \ldots, i_{n}\right)} f_{i_{1}, \ldots, i_{n}} u_{1}^{i_{1}} \ldots u_{n}^{i_{n}}, \quad f_{i_{1}, \ldots, i_{n}} \in k,
$$

where we identify $f$ with an element of the field $k\left(\left(u_{1}\right)\right) \ldots\left(\left(u_{n}\right)\right)$. Here $K((t))$ denotes the field of the Laurent power series in $t$ with coefficients in a field $K$.

Definition A.3. Let $\omega$ be a rational $n$-form on $X$. Fix a complete flag $F$ of irreducible subvarieties (A1). Parshin's residue $\operatorname{res}_{F} \omega$ at the flag $F$ is the number $f_{-1, \ldots,-1}$ constructed above.

Parshin's residue does not depend on the choice of local parameters $\left(u_{1}, \ldots, u_{n}\right)_{F}$. The proof of this statement is similar to the proof we gave for the invariance of the toroidal residue in Proposition 4.2.

Example 2. Let $f_{1}, \ldots, f_{n}$ be rational functions on an algebraic variety $X$, whose zero loci $\left\{f_{i}=0\right\}$ intersect transversely at a non-singular point $x \in X$. Denote by $Z_{i}$ the irreducible component of $\left\{f_{i}=0\right\}$ that contains $x$.

Let $f_{0}$ be any rational function on $X$ which is regular in an open neighborhood of $x$. Then

$$
\operatorname{res}_{F} f_{0} \frac{d f_{1}}{f_{1}} \wedge \cdots \wedge \frac{d f_{n}}{f_{n}}=\mu(x) f_{0}(x),
$$

where $F: x=X_{0} \subset X_{1} \subset \cdots \subset X_{n-1} \subset X$, for $X_{i}=Z_{i+1} \cap \cdots \cap Z_{n}$, and $\mu(x)=\mu_{1} \ldots \mu_{n}$ is the product of the multiplicities $\mu_{i}$ of $f_{i}$ along $Z_{i}$.

Indeed, for the system of local parameters at $F$ we can choose the local equations $u_{i}$ of $Z_{i}$ at $x$. Then

$$
\operatorname{res}_{F} f_{0} \frac{d f_{1}}{f_{1}} \wedge \cdots \wedge \frac{d f_{n}}{f_{n}}=\operatorname{res}_{F} f_{0} \frac{d u_{1}^{\mu_{1}}}{u_{1}^{\mu_{1}}} \wedge \cdots \wedge \frac{d u_{n}^{\mu_{n}}}{u_{n}^{\mu_{n}}}=\operatorname{res}_{F} \mu(x) f_{0} \frac{d u_{1}}{u_{1}} \wedge \cdots \wedge \frac{d u_{n}}{u_{n}}=\mu(x) f_{0}(x) .
$$

\section{A.3 Reciprocity laws}

Now we will formulate Parshin's reciprocity laws for the tame symbol and the residue.

Theorem A.4. Let $X$ be a complete irreducible $n$-dimensional algebraic variety over an algebraically closed field $k$. Fix a partial flag of irreducible subvarieties $X_{0} \subset \cdots \subset \widehat{X}_{i} \subset \cdots \subset X_{n}=X$, where $X_{i}$ is omitted. Then

i) for any $n+1$ rational functions $f_{0}, \ldots, f_{n}$ on $X$

$$
\prod_{X_{i}}\left\langle f_{0}, \ldots, f_{n}\right\rangle_{X_{0} \subset \cdots \subset X_{i} \subset \cdots \subset X_{n}}=1
$$

ii) for any rational $n$-form $\omega$ on $X$

$$
\sum_{X_{i}} \operatorname{res}_{X_{0} \subset \cdots \subset X_{i} \subset \cdots \subset X_{n}} \omega=0
$$

where the product (sum) is taken over all irreducible subvarieties $X_{i}$ that complete the flag, and is finite.

It follows by definition that Parshin's symbol $\left\langle f_{0}, \ldots, f_{n}\right\rangle_{F}$ equals 1 unless $F$ consists of intersections of components of the divisors of $f_{0}, \ldots, f_{n}$. This shows that the above product is finite. Similarly, Parshin's residue $\operatorname{res}_{F} \omega$ is zero unless $F$ consists of intersections of components of the polar set of $\omega$, hence, the above sum is finite.

In the proof of the main theorems (Theorems 3.15 and 4.8) we referred to the special case of the reciprocity law when $i=0$. In this case the proof of the first part of Theorem A.4 is based on 


\section{RESIDUES AND TAME SYMBOLS ON TOROIDAL VARIETIES}

the 'reduction formula' for the symbol. Namely, the property of the symbol analogous to the cofactor expansion for the determinant allows one to represent the $n$-dimensional symbol as a product of symbols of dimension $n-1$ (see [FP04]). Then the statement follows from Weil's reciprocity by induction.

For the case of the residue, notice that the residue of the $n$-form $\omega$ at the flag $F$ is equal to the sum of the residues of 1 -forms $\omega_{1}$ on $X_{1}$ at $X_{0}$. The statement then follows from the one-dimensional residue formula.

\section{REFERENCES}

Ber75 D. N. Bernstein, The number of roots of a system of equations, Funct. Anal. Appl. 9 (1975), 183-185.

BM96 J.-L. Brylinski and D. A. McLaughlin, Multidimensional reciprocity laws, J. reine angew. Math. 481 (1996), 125-147.

BZ88 Y. D. Burago and V. A. Zalgaller, Geometric inequalities, Grundlehren Math. Wiss., vol. 285 (Springer, Berlin, 1988).

CCD97 E. Cattani, D. Cox and A. Dickenstein, Residues in toric varieties, Compositio Math. 108 (1997), $35-76$.

CD97 E. Cattani and A. Dickenstein, A global view of residues in the torus, J. Pure Appl. Algebra 117/118 (1997), 119-144.

CDS98 E. Cattani, A. Dickenstein and B. Sturmfels, Residues and resultants, J. Math. Sci. Univ. Tokyo 5 (1998), 119-148.

Cox96 D. Cox, Toric residues, Ark. Mat. 34 (1996), 73-96.

FP04 T. Fimmel and A. N. Parshin, An introduction to the higher adelic theory, book in preparation.

Ful93 W. Fulton, Introduction to toric varieties (Princeton University Press, Princeton, NJ, 1993).

Gel96 O. A. Gel'fond, Combinatorial coefficients and the mixed volume of polytopes, Funct. Anal. Appl. 30 (1996), 207-208.

GK96 O. A. Gel'fond and A. G. Khovanskii, Newton polyhedra and Grothendieck residues, Dokl. Akad. Nauk 350 (1996), 298-300 (in Russian).

GK02 O. A. Gel'fond and A. G. Khovanskii, Toric geometry and Grothendieck residues, Mosc. Math. J. 2 (2002), 99-112.

Dan78 V. I. Danilov, The geometry of toric varieties, Russian Math. Surveys 33 (1978), 97-154.

Kho99 A. G. Khovanskii, Newton polyhedra, a new formula for mixed volume, product of roots of a system of equations, Fields Inst. Commun. 24 (1999), 325-364.

KKMS73 G. Kempf, F. Knudsen, D. Mumford and B. Saint-Donat, Toroidal embeddings I, Lecture Notes in Mathematics, vol. 339 (Springer, Berlin, 1973).

Par75 A. N. Parshin, Class fields and algebraic K-theory, Uspekhi Mat. Nauk 30 (1975), 253-254.

Par83 A. N. Parshin, Chern classes, adeles and L-functions, J. reine angew. Math., 341 (1983), 174-192.

PS93 P. Pedersen and B. Sturmfels, Product formulas for resultants and Chow forms, Math. Z. 214 (1993), 377-396.

Ser88 J.-P. Serre, Algebraic groups and class fields, Graduate Texts in Mathematics, vol. 117 (Springer, Berlin, 1988).

Sop02 I. Soprounov, Parshin's symbols and residues, and Newton polyhedra, PhD thesis, University of Toronto (2002).

Ivan Soprounov isoprou@math.umass.edu

Department of Mathematics and Statistics, University of Massachusetts Amherst, Amherst, MA 01003, USA 